\title{
Hepatocarcinoma and Non Alcoholic Fatty Liver Disease
}

\begin{abstract}
Ana Luisa Viera Rodrigues de Queiroz ${ }^{1}$, Dora Gama Riberio Leite Altikes ${ }^{1}$, Daniele Camargo Pinheiro Alves ${ }^{2}$, Paula Bechara Poletti ${ }^{1}$, Luiza Alencar Saldanha Queiroz ${ }^{1}$, Cláudia De Fátima Gomes Vieira De Oliveira ${ }^{1}$, Camila Toledo Turano ${ }^{1}$, Leonardo Renna de Azevedo ${ }^{1}$, Mayra Martins Cruz ${ }^{1}$, Erika Nogueira Loures ${ }^{1}$, Julia Vieira Kuster $^{1}$, Leticia Cristina de Araujo Diaz Vazquez ${ }^{1}$ and Rogério Camargo Pinheiro Alves ${ }^{1 *}$
\end{abstract}

${ }^{1}$ Gastroenterolgy and Hepatology Department, Hospital do Servidor Publico Estadual de São Paulo, Brazil

${ }^{2}$ Clinical Oncology Department, Hospital do Servidor Publico Estadual de São Paulo, Brazil

Submission: November 25, 2019; Published: December 09, 2019

*Corresponding author: Rogério Camargo Pinheiro Alves, Gastroenterology and Hepatology Department, Hospital do Servidor Publico Estadual de São Paulo, Brazil

\begin{abstract}
Non-alcoholic Fatty liver disease (NAFLD) incidence is rising worldwide due to the epidemic of obesity and diabetes and it is becoming an important etiology of Hepatocellular carcinoma (HCC), which is the most frequent primary liver cancer, the fourth most common malignancy neoplasm and it's deaths are in third place among Cancer-related deaths. Many NAFLD patients are diagnosed with HCC in the late stage of the disease, with large tumors, giving them a bad prognosis. This occurs mainly because of the lack of screening programs in these population.

Not all of the factors involved in the disease development have been elucidated but some genetic factors have been implicated in HCC emergence. Many factors, like tumor size and patients' comorbidities, influence HCC treatment in NAFLD patients, making it more difficult than in other etiologies. In this article, we will review the epidemiology, pathogenies and diagnosis of HCC linked to NAFLD.

Keywords: Hepatocellular carcinoma; Steatosis; NAFLD; Liver cancer; NASH; Cirrhosis; Hyperinsulinemia; Insulin resistance; Steatosis; Oxidative stress

Abbrevations: NAFLD: Non-Alcoholic Fatty Liver Disease; HCC: Hepatocellular Carcinoma; PNPLA3: Phospholipase Protein 3; ER: Endoplasmic Reticulum; ROS: Reactive Oxygen Species; TNF: Tumor Necrosis Factor; TNFSF: TNF Superfamily Members; LSECs: Liver Sinus Endothelial Cells; mTOR: Mammalian Target of Rapamycin
\end{abstract}

\section{Introduction}

Hepatocellular carcinoma (HCC) is the most common primary liver malignant tumor as well as the fourth most incident neoplasm in the world. Some cases are estimated to happen in the absence of cirrhosis [1]. Non-alcaholic fatty liver disease (NAFLD) is the most common chronic liver disease in western world, with a prevalence in the world population around $20-30 \%$. It is an important risk factor to develop CHC and the spectrum of NAFLD ranges from steatosis to steatohepatitis and cirrhosis [2-4].

Patients with nonalcoholic steatohepatitis (NASH) have a higher risk of developing cancer due to some conditions associated with the disease, such as hyperinsulinemia, insulin resistance, steatosis, oxidative stress, and chronic inflammation adipokine / cytokine balance [5]. Thus, it plays an important role in liver carcinogenesis, promoting cell growth and DNA damage [1]. A meta-analysis showed a $17 \%$ increase in HCC in the overweight population and $90 \%$ in obese women compared to regular weight population [6].

\section{Epidemiology}

HCC accounts for over 700,000 new cases and 600,000 deaths per year. It accounts for about $9.2 \%$ of new tumor cases. It occurs more often in men than women, with a ratio of $2: 1-4: 1$. Most cases occur between 30 and 60 years of age [7]. The occurrence has increased in recent decades in rich countries (from 1.4 per 100,000 in $1975-1977$ to 4.8 per 100,000 in $2005-2008$ ) [8]. In Hispanics, the NASH-HCC ratio has a higher risk, affecting 12.3 per 1000 people a year [9]. In Western countries, cases of HCC and viruses are decreasing due to hepatitis $B$ vaccination and 
effective treatments for viral hepatitis. In addition, cases of alcoholic etiology are also going through a relative decrease [10].

In the United States, the third most common etiology of HCC is NAFLD due to the large number of patients with this condition [11]. Due to the obesity epidemic, NAFLD-related HCC has a growth of $9 \%$ per year, with the highest cancer growth rate [12]. In addition, diabetes and obesity have a positive association with HCC with odds ratio of 4.33 (95\% CI 1.89-9.86) and 1.97 (95\% CI 1.03-3.79), respectively [13]. The presence of a single-nucleotide genetic polymorphism of the domain-containing phospholipase protein 3 (PNPLA3) or rs738409 has a higher risk of HCC-NASH [9].

Patients with HCC and NASH have a shorter survival and often die more from primary liver cancer than other patients with HCC of other etiologies [11]. This is probably due to the larger tumor size, the older age of the patient and the later and more advanced diagnosis of the tumor [14]. In a retrospective cohort of 1,500 Americans, about $13 \%$ of hepatocellular carcinoma cases occurred in the absence of cirrhosis, the largest risk factor for this being non-alcoholic fatty liver disease [15]. About $3-15 \%$ of NASH patients progress to cirrhosis and about $4-27 \%$ of these progress to HCC [16].

Post liver transplantation, risk factors such as diabetes, obesity, dyslipidemia and cardiovascular disease are more common. However, short to medium term survival does not differ from other etiologies [17]. The prevalence of liver transplantation due to NASH cirrhosis has been increasing mainly due to the high prevalence of obesity and diabetes mellitus. In the USA, from 2001 to 2009 , the percentage of transplants for this cause increased from $1.2 \%$ to $9.7 \%$ [18]. Thus, it is already the fastest growing etiology of cirrhosis and the main indication for transplantation in the USA [9]. The maintenance of smoking is common and the occurrence of cardiovascular diseases is higher than in other etiologies [18].

\section{NASH and HCC Pathophysiology}

The pathogenesis of HCC associated with NAFLD was described by the concept of the "two-hit hypothesis", but this concept was not sufficient to show the various molecular and metabolic implications of disease progression. Currently, the "parallel multiple hit hypothesis" is being used to explain the progression of HCC in NAFLD [19,20].

The pathophysiology is composed off the involvement of several mechanisms such as: immune and inflammatory responses, DNA damage, oxidative stress, autophagy and the microbiome [21]. Several mechanisms through the TNF-centered inflammatory pathway may lead to the progression of HCC in NAFLD. The high fat diet supports stress of the endoplasmic reticulum (ER) that promotes synthesis and storage of liver fat and steatosis. ER stress and steatosis increase the production of reactive oxygen species (ROS) to cause oxidative stress and alteration in cell DNA which increase local inflammatory microenvironment. Inflammatory cells, like macrophages and linfocito $\mathrm{T}$ produce, mainly, Tumor necrosis factor (TNF) and interleucines $1,6,10$. Those refeed the inflammatory microenvironment, factors stimulating hepatocyte proliferation and HCC expansion [22,23].

Many factors contribute to the development of HCC in NAFLD patients. Increased levels of TNF superfamily members (TNFSF), transforming growth factor $\beta$ (TGF $\beta$ ) and IL-18, together with chronic hepatocyte cell death and compensatory proliferation increase the risk of hepatocellular carcinoma (HCC). Besides that, hepatic stellate cells (HSCs) activation, liver sinus endothelial cells (LSECs) and chronic liver proliferation in a chronic regenerative environment, accompanied by chromosomal aberrations, contribute to the development of HCC. [21].

Autophagy is a catabolic cellular process that results in the degradation of cell components using lysosomes. This degradation is dependent on autophagy-related proteins and occurs selectively, marking the charges with molecular markers with ubiqutin. In NAFLD, excess triglycerides and FFA suppress the onset of autophagy by activating the mammalian target of rapamycin (mTOR) and suppressing serine / threonine protein kinase and ULK1, leading to increased hepatic oxidative stress [24,25].

Metabolic stress causes autophagy tumor cells to accumulate p62 (an autophagy substrate), resulting in retention of damaged mitochondria, increasing oxidative stress and activation of DNA damage response. In addition, a late-stage autophagy suppressor (Rubicon) is elevated in NAFLD and is responsible for accelerating hepatocellular apoptosis and accumulation [26]. Other factor to contributing to development of HCC is dysbiosis, which is the imbalance of the intestinal bacterial flora. Studies suggest that dysbiosis may be responsible for metabolic changes and may lead to the generation of free radicals, contributing to the inflammatory cascade contributing to the generation of liver disease and chronic liver diseases such as NAFLD, NASH and alcoholic liver disease [21]

The effects of dysbiosis on hepatic fibrosis and carcinogenesis through the microbiota-liver axis occur through various factors derived from the gut. Due to the anatomical position of the liver, intestinal bacteria and pathogen-associated molecular patterns (PAMPs) have a major impact on portal vein liver disease [22]. NAFLD patients have a significant increase in peripheral blood endotoxin concentration, intestinal permeability, intestinal bacterial overgrowth, and portal blood lipopolysaccharide detection, which increase TNF- $\alpha$ production in Kupffer cells by enhancing receptor-type signal. Toll (TLR4). The increased sensitivity of LPS to growth transforming factor beta (TGF- $\beta$ ) results in progression towards hepatic fibrosis and the promotion of liver carcinogenesis [22]. 


\section{Diagnosis}

Ultrasound is the most cost-effective screening method for HCC recommended by societies [27]. However, in patients with central obesity and high BMI this method has limitations. Thus, its sensitivity to detect tumors smaller than $3 \mathrm{~cm}$ is greatly reduced at $\mathrm{BMI}>35$ [28]. Magnetic resonance imaging is more sensitive to detect possibly healing lesions and it can be used in obese patients with NASH cirrhosis who are candidates for curative treatments (CHILD A, good status) [27]. NAFLD-associated HCC and other HCC etiologies are similarly approached by the European and American guidelines [21]. The guidelines suggest screening only the population with cirrhosis, but patients with steatosis and mild steatohepatitis should have be given attention as well, since there are cases of CHC reported in this population, even without cirrhosis.

One study evaluated MRI scans of 21 patients with HCC and NASH. Eighteen of them were men and 3 were women with a mean age of $67.9 \pm 10.2$ (SD) years (range: 36-85 years). The images were analyzed qualitatively and quantitatively. The study noted that $57 \%$ of HCC in NASH may be presented as a lesion smaller than $20 \mathrm{~mm}$ and $40 \%$ does not have wash in and wash out, suggesting that the classical imaging criteria developed for the noninvasive diagnosis of HCC should be applied with caution in patients with NASH [29].

\section{Conclusion}

The incidence of nonalcoholic fatty liver disease is increasing in the world and has reached the leading cause of chronic liver disease. Eventually it will be the leading etiology of HCC. Thus, its prevention with measures aimed at weight loss and lifestyle changes is important. The pathogenic mechanisms are being unraveled. In addition, screening is needed in this population in order to attempt an earlier diagnosis. A more effective tool and a few more data will be necessary to select the higher risk population who will need to be submitted to a screening program with cost effectiveness. Identifying biomarkers is an important goal to improve treatment strategies.

\section{References}

1. Petta S, Craxì A (2010) Hepatocellular carcinoma and non-alcoholic fatty liver disease: from a clinical to a molecular association. Curr Pharm Des 16(6): 741-752.

2. Loomba R, Adams LA (2019) The 20\% Rule of NASH Progression: The Natural History of Advanced Fibrosis and Cirrhosis due to NASH. Hepatology 70(6): 1885-1888.

3. Ching-Yeung Yu B, Kwok D, Wong VW (2019) Magnitude of Nonalcoholic Fatty Liver Disease: Eastern Perspective. J Clin Exp Hepatol 9(4): 491496.

4. Reddy SK, Steel JL, Chen HW, DeMateo DJ, Cardinal J, et al. (2012) Outcomes of Curative Treatment for Hepatocellular Cancer in Nonalcoholic Steatohepatitis Versus Hepatitis C and Alcoholic Liver disease. Hepatology 55(6): 1809-1819.
5. Giovannucci E, Harlan DM, Archer MC, Bergenstal RM, Gapstur SM, et al. (2010) Diabetes and cancer: aconsensus report. Diabetes Care 33(7): 1674-1685.

6. Larsson SC, Wolk A (2007) Overweight, obesity and risk of liver cancer: a meta-analysis of cohort studies. Br J Cancer 97(7): 1005-1008.

7. Jemal A, Bray F, Center MM, Ferlay J, Ward E, et al. (2011) Global cancer statistics. CA Cancer J Clin 61(2): 69-90.

8. Baffy G, Brunt EM, Caldwell SH (2012) Hepatocellular carcinoma in non-alcoholic fatty liver disease: an emerging menace. J Hepatol 56(6): 1384-1391.

9. Kim HS, El-Serag HB (2019) The Epidemiology of Hepatocellular Carcinoma in the USA. Curr Gastroenterol Rep 21(4): 17.

10. Sagnelli E, Macera M, Russo A, Coppola N, Sagnelli C (2019) Epidemiological and etiological variations in hepatocellular carcinoma. Infection.

11. Mohamad B, Shah V, Onyshchenko M, Elshamy M, Aucejo F, et al. (2016) Characterization of hepatocellular carcinoma (HCC) in non-alcoholic fatty liver disease (NAFLD) patients without cirrhosis. Hepatol Int 10(4): 632-639.

12. Younossi ZM, Otgonsuren M, Henry, Venkatesan C, Mishra A, et al. (2015) Association of nonalcoholic fatty liver disease (NAFLD) with hepatocellular carcinoma (HCC) in the United States from 2004 to 2009. Hepatology 62(6): 1723-1730.

13. Turati F, Talamini R, Pelucchi C, Polesel J, Franceschi S, et al. (2013) Metabolic syndrome and hepatocellular carcinoma risk. Br J Cancer 108(1): 222-228.

14. Calzadilla Bertot L, Adams LA (2016) The natural course of nonalcoholic fatty liver disease. Int J Mol Sci 17(05): 774.

15. Mittal S, El-Serag HB, Sada YH, Kanwal F, Duan Z, et al. (2016) Hepatocellular carcinoma in the absence of cirrhosis in United States veterans is associated with nonalcoholic fatty liver disease. Clin Gastroenterol Hepatol 14(1): 124-131.

16. Dhamija E, Paul SB, Kedia S (2019) Non-alcoholic fatty liver disease associated with hepatocellular carcinoma: An increasing concern. Indian J Med Res 149(1): 9-17.

17.Ângulo P (2006) Nonalcoholic fatty liver disease and liver transplantation Liver Transpl 12: 523-534.

18. Castelló B, Aguilera V, Blázquez MT, Rubín Á, García M, et al. (2019) Post-transplantation outcome in non-alcoholic steatohepatiti scirrhosis: Comparison with alcoholic cirrhosis. Ann of Hepatol 18(6): 855-861.

19. Day CP, James OF (1998) Steatohepatitis: a tale of two "hits"? Gastroenterology 114(4): 842-845.

20. Tilg H, Moschen AR (2010) Evolution of inflammation in nonalcoholic fatty liver disease: the multiple parallel hits hypothesis. Hepatology 52(5): 1836-1846.

21. Anstee QM, Reeves HL, Kotsiliti E, Govaere O, Heikenwalder M (2019) From NASH to HCC: current concepts and future challenges. Nat Rev Gastroenterol Hepatol 16(7): 411-428.

22. Takakura K, Oikawa T, Nakano M, Saeki C, Torisu Y, et al. (2019) Recent insights into the multiple pathways driving nonalcoholic steatohepatitis-derived hepatocellular carcinoma. Front Oncol 9: 762.

23. Ringelhan M, Pfister D, O’Connor T, Pikarsky E, Heikenwalder M (2018) The immunology of hepatocellular carcinoma. Nat Immunol 19(3): 222-232. 
24. Khaminets A, Behl C, Dikic I (2016) Ubiquitin- dependent and independent signals in selective autophagy. Trends Cell Biol 26(1): 6-16.

25. Kim J, Kundu M, Viollet B, Guan KL (2011) AMPK and mTOR regulate autophagy through direct phosphorylation of Ulk1. Nat Cell Biol 13(2): 132-141.

26. Tanaka S, Hikita H, Tatsumi T, Sakamori R, Nozaki Y, et al. (2016) Rubicon inhibits autophagy and accelerates hepatocyte apoptosis and lipid accumulation in nonalcoholic fatty liver disease in mice. Hepatology 64(6): 1994-2014.
27. EASL Clinical Practice Guidelines (2018) Management of hepatocellular carcinoma. J Hepatol 69(01): 182-236.

28. Wong LL (2017) Pitfalls in surveillance for hepatocellular carcinoma: how successful is it in the real world? Clin Mol Hepatol 23(03): 239248.

29. Al-Sharhan F, Dohan A, Barat M, Feddal A, Terris B, et al. (2019) MRI presentation of hepatocellular carcinoma in non-alcoholic steatohepatitis (NASH). Eur J Radiol 119: 108648.

This work is licensed under Creative Commons Attribution 4.0 License

DOI: $10.19080 / A R G H .2019 .14 .555885$ 